\title{
PERMUTATIONS IN A FINITE FIELD
}

\section{CARLITZ}

A polynomial $f(x)$ with coefficients $\in G F(q)$ is called a permutation polynomial if the numbers $f(\alpha)$, where $\alpha \in G F(q)$, are a permutation of the $\alpha$ 's. (For references see [2, Chap. 18].) In a letter to the writer, E. G. Straus has inquired whether all permutation polynomials can be generated by means of the special types

$$
\alpha x+\beta, \quad x^{q-2} \quad(\alpha, \beta \in G F(q), \alpha \neq 0) .
$$

For $q=5$, this was proved to be true by Betti; for $q=7$ the corresponding result was verified by Dickson [1, p. 119].

In this note we show very simply that this result holds for all $q$. Since the totality of permutation polynomials evidently furnishes a representation of the symmetric group on $q$ letters, it will suffice to show that every transposition $(0 \alpha)$ can be generated by means of the special polynomials (1); here $\alpha$ denotes a fixed nonzero number $\in G F(q)$. We consider the following polynomial

$$
g(x)=-\alpha^{2}\left(\left((x-\alpha)^{q-2}+\frac{1}{\alpha}\right)^{q-2}-\alpha\right)^{q-2} .
$$

Then in the first place we easily verify that $g(0)=\alpha$ and $g(\alpha)=0$. Secondly if $\beta \neq 0, \beta \neq \alpha$, then

$$
\begin{aligned}
g(\beta) & =-\alpha^{2}\left(\left(\frac{1}{\beta-\alpha}+\frac{1}{\alpha}\right)^{q-2}-\alpha\right)^{q-2} \\
& =-\alpha^{2}\left(-\frac{\alpha^{2}}{\beta}\right)^{q-2}=\beta,
\end{aligned}
$$

so that $\beta$ is carried into itself. This shows that the polynomial (2) does indeed effect the transposition $(0 \alpha)$, and therefore our result follows.

We may state the following

THEOREM. Every permutation on the numbers of $G F(q)$ can be derived from (1).

\section{REFERENCES}

1. L. E. Dickson, The analytic representation of substitutions on a power of a prime number of letters with a discussion of the linear group, Ann. of Math. vol. 11 (1896-97) pp. $65-120$.

2. - History of the theory of numbers, vol. 3, Washington, 1923.

DUKE UNIVERSITY

Received by the editors December 6, 1952. 